Published in final edited form as:

Alcohol. 2006 August ; 40(1): 61-65.

\title{
Proceedings of the 2006 Annual Meeting of the Fetal Alcohol Spectrum Disorders Study Group
}

\author{
Daniel J. Bonthius, MD, $\mathrm{PhD}^{1}$, Heather Carmichael Olson, $\mathrm{PhD}^{2}$, and Jennifer D. Thomas, \\ $\mathrm{PhD}^{3}$ \\ 1 Department of Pediatrics, University of Iowa College of Medicine, Iowa City, IA \\ 2 Children's Hospital \& Regional Medical Center, Families Moving Forward Program, Seattle, WA \\ 3 Center for Behavioral Teratology, Dept. of Psychology, San Diego State University, San Diego, CA
}

\begin{abstract}
This article describes the proceedings of the 2006 Annual Meeting of the Fetal Alcohol Spectrum Disorders Study Group (FASDSG), which was held in Baltimore, Maryland on June 24, 2006. The meeting was held in conjunction with the annual meeting of the Research Society on Alcoholism and was supported by a grant from the National Institute on Alcohol Abuse and Alcoholism. The 2005-2006 FASDSG officers, Daniel J. Bonthius (president), Heather Carmichael Olson (vicepresident), and Jennifer Thomas (secretary-treasurer), organized the meeting. Nationally prominent speakers delivered plenary lectures on topics of newborn screening, ethics, and neuroscience. Selected members of the FASDSG provided brief scientific data (FASt) reports, describing new research findings. Representatives from national agencies involved in fetal alcohol syndrome (FAS) research, treatment, and prevention provided updates regarding priorities, funding, and agency activities. Presentations were also made by the 2006 Student Merit Award recipient and by the 2006 Rosett Award recipient. The meeting served as a forum for clinicians, neuroscientists, psychologists, social scientists and other professionals to discuss recent advances in FAS research and to identify the most important gaps in the understanding of alcohol-induced teratology.
\end{abstract}

\section{Keywords}

Fetal Alcohol Syndrome; Fetal Alcohol Spectrum Disorders; Ethanol; Prenatal; Teratology

\begin{abstract}
The Fetal Alcohol Spectrum Disorders Study Group (FASDSG) met for their annual meeting on June 24, 2006 in Baltimore, Maryland. The meeting was supported by a grant from the National Institute on Alcohol Abuse and Alcoholism. The program included guest lectures by nationally prominent speakers in fields of neuroscience, medicine, and ethics; research updates from a variety of laboratories across North America; funding and activity updates from national agencies involved in fetal alcohol syndrome (FAS) research, treatment and prevention; and presentation of awards for achievement and devotion to FASD research. The FASDSG meeting was held in conjunction with the annual meeting of the Research Society on Alcoholism (RSA).
\end{abstract}

Please address all correspondence to: Daniel J. Bonthius, MD, PhD, Division of Child Neurology, Department of Pediatrics, 2504 JCP, University of Iowa Hospital, 200 Hawkins Drive, Iowa City, Iowa 52242, Telephone: (319) 356-7727, FAX: (319) 356-4855, Email: daniel-bonthius@uiowa.edu.

Publisher's Disclaimer: This is a PDF file of an unedited manuscript that has been accepted for publication. As a service to our customers we are providing this early version of the manuscript. The manuscript will undergo copyediting, typesetting, and review of the resulting proof before it is published in its final citable form. Please note that during the production process errorsmaybe discovered which could affect the content, and all legal disclaimers that apply to the journal pertain. 
Daniel Bonthius, MD, PhD was the presiding officer. Heather Carmichael Olson, $\mathrm{PhD}$ and Jennifer Thomas, PhD served as vice-president and secretary-treasurer, respectively.

The first plenary lecture was delivered by Elizabeth Thomson, DNSc, Program Director for Ethical, Legal, and Social Implications Research at the National Human Genome Research Institute. In her address, entitled "Ethical, legal and social implications of genetics and genomics," Dr. Thomson described the goals and successes of the Human Genome Project (HGP) and outlined the many concerns and issues associated with that national endeavor. She explained that the ethical, legal and social implications (ELSI) research program has been a critical component of the U.S. human genome project since the program's inception in 1990 and that five percent of HGP funds are devoted to ELSI research. Dr. Thomson stated that the aims of the ELSI program are to ensure that 1) genetic research is conducted in an ethically sound manner, 2) genetic technologies are integrated appropriately into clinical and nonclinical settings, 3) genetic information is correctly interpreted and appropriately used and 4) health professionals and the public become more genetically literate.

Dr. Thomson outlined research issues that need further consideration and deliberation. She explained that the greatest challenge in genetics and genomics research is to protect participant privacy, when there is also a desire to place genomic and health outcome information into publicly accessible databases and a desire to link back to the donor for follow-up information. Dr. Thomson described the forces that often drive the development of new genetic tests as well as the potential benefits and risks of genetic testing. She provided examples of ways in which genetic testing has been misused and misinterpreted and the consequences of this misuse. Dr. Thomson stated that genetics and genomics will become increasingly interwoven in social policy issues. In closing, she stressed that genetic information is not deterministic and that people and their health should not be reduced to their genetic make-up.

The second plenary lecture was delivered by James W. Hanson, MD, Director of the Center for Developmental Biology and Perinatal Medicine at the National Institute of Child Health and Human Development. Dr. Hanson's address was entitled "Expanded newborn screening: national goals and implementation initiatives. How might this model relate to the prevention of FAS?" In the first half of his address, Dr. Hanson described success stories in the prevention and treatment of birth defects and genetic disorders. He pointed to the identification of thalidomide and alcohol as teratogens, the treatment of folate deficiency, and immunization against rubella as important steps in the reduction of birth defects. Dr. Hanson then described the basis and history of newborn screening. He defined rational newborn screening as a systematic search for serious conditions that are otherwise usually missed prior to adverse consequences. He explained that interventions to improve outcomes should be available and that many screening approaches, besides laboratory testing, can be used. Dr. Hanson stated that newborn screening began in the 1960s, that it has gradually expanded with great variability, that the expansion has depended on technological innovations, and that newborn screening has been poorly understood by healthcare providers and the public.

In the second half of his address, Dr. Hanson discussed newborn screening as a model approach to the prevention and treatment of fetal alcohol syndrome and related effects. He discussed the resources, knowledge and values issues that need to be addressed. In addition, he discussed the needs and priorities to permit FASD (fetal alcohol spectrum disorders) and related outcomes research to be developed or expanded in other countries. Finally, Dr. Hanson discussed the implications and prospects of newborn screening for FASD. He stated that, although FASD presents a valid public health target for prevention, several barriers currently preclude effective implementation. He explained that inadequate biomarkers of significant prenatal alcohol exposure seriously limit effective newborn screening for FASD and suggested that prenatal screening may be more useful. Furthermore, he stated that lack of an effective treatment is a 
major shortcoming that dampens enthusiasm for postnatal screening for FASD. He emphasized, however, that technological advancements and treatment breakthroughs can change the balance of equations regarding the rationale for newborn screening for individual disorders and that such advancements in alcohol research could make FASD an attractive target for newborn screening.

The FASDSG Presidential Lecture was delivered by Michael Dailey, PhD, Associate Professor of Biological Sciences at the University of Iowa. In his lecture, entitled "Microglia on the move: the dynamics of microglial activation in injured neonatal brain tissues," Dr. Dailey described the processes by which microglia respond to neuronal injury, the cell-cell signals that induce microglial responses to injury, and the intracellular mechanisms that mediate microglial responses. Utilizing state-of-the-art staining and imaging techniques, including vital staining of microglia, confocal 3-D reconstruction, and time-lapse confocal imaging, Dr. Dailey demonstrated changes in microglial morphology and behavior during activation, including retraction of branches and initiation of motility. He further demonstrated the homing of microglia to injured neurons and the phagocytosis of dead neuronal tissue.

Dr. Dailey utilized pharmacological agents to identify the extracellular signals that guide microglia to regions of neuronal injury. Through the use of apyrase, an enzyme that degrades extracellular ATP/ADP, Dr. Dailey demonstrated the critical role of ATP/ADP in inducing microglial branch extension and directed cellular migration. He further demonstrated that the chemotaxic migration of microglia, induced by ATP/ADP, is mediated by the P2Y12 receptor on the microglial cellular surface. In addition, Dr. Dailey discussed important developmental changes in microglial responses. He demonstrated that neonatal and mature microglia differ markedly in branch length, motility, locomotion and homing. He ended his talk by pointing out that alcohol can substantially alter microglial responses and that microglia may play important roles in the pathogenesis of FASD.

At its annual meeting, the FASDSG receives a yearly update from representatives of federal agencies that provide funding for FASD research or that are deeply involved in issues involving individuals with FASD. Dr. Kenneth Warren, representing the National Institute on Alcohol Abuse and Alcoholism (NIAAA), discussed that agency's five year strategic plan for FASDrelated research. Dr. Sally Anderson, representing the Interagency Coordinating Committee on FAS (ICCFAS), discussed the purpose and goals of the ICCFAS, the multiple agencies with membership on the committee, the themes around which the committee bases its work, and its recent activities. Dr. Sherry Ceperich represented the National Center on Birth Defects and Developmental Disabilities at the Centers for Disease Control and Prevention. She described the surveillance systems, epidemiological studies, and prevention programs sponsored by the CDC aimed at reducing the number and severity of alcohol-exposed pregnancies. Finally, Ms. Aimee Bonsu and Dr. Callie Gass, representing the Substance Abuse and Mental Health Services Administration (SAMHSA), described the SAMHSA FASD Center for Excellence and highlighted the FASD-related resources that the center has created for communities, families and professionals.

Each year at its annual meeting, the Fetal Alcohol Spectrum Disorders Study Group provides the opportunity for some of its members to briefly present their newest data. By convention, the presentations are limited to a single slide, five minutes of verbal presentation, and one minute of questions and answers. These sessions, which are an opportunity for new investigators to present their research interests and for established investigators to highlight their most important recent findings, are referred to as the "FASt sessions".

More abstracts were submitted than time allowed for presentation at the 2006 meeting. Therefore, abstracts were selected by the FASDSG officers based on merit, with an attempt to 
balance the presentations among the research interests of the group. Presentations that focused on basic science research and human research were selected for presentation in proportion to the number of abstracts submitted in each category.

\section{In the basic science research category}

S. Shah and T.A. Lindsley investigated the effects of ethanol on F-actin organization in axonal growth cones. Using primary cultures of embryonic rat hippocampal neurons, they found that ethanol substantially rearranges the F-actin cytoskeleton in axonal growth cones. This work may shed important light on the mechanism by which ethanol disrupts motile events, including neuron migration, process outgrowth, and axon guidance, in the developing brain.

K.K. Caldwell, R.D. Paz, M.H. Laughlin, S.L. Ruiz, F.T Candalaria and A.M. Allen assessed the relationship between prenatal alcohol exposure, depression, brain-derived neurotrophic factor (BDNF) levels, and histone $\mathrm{H} 4$ acetylation using a mouse model of prenatal ethanol exposure. They found that ethanol-exposed offspring displayed increased learned helplessness behavior and reductions in BDNF protein levels and histone $\mathrm{H} 4$ acetylation in the medial frontal cortex. These findings confirm a relationship between prenatal ethanol exposure and depression and identify BDNF and histone $\mathrm{H} 4$ as possible targets for therapeutic intervention in the treatment of prenatal ethanol exposure-linked depression.

E.A. Myers, S. Parnell, G.A. Johnson, B. Guenther, R. Herbert, M.A. Styner, and K.K. Sulik utilized high resolution magnetic resonance imaging (MRI) to study alcohol-induced CNS abnormalities in fetal mice. Using state-of-the-art imaging technology, they found marked enlargement of forebrain ventricles and reductions in hindbrain ventricles in ethanol-exposed fetal mice. In addition, they found that facial dysmorphology corresponded to the size and shape of the forebrain. They concluded that the new MRI technology allows comprehensive studies that would be prohibitively labor intensive with conventional approaches.

S.L. Ruiz, C.F. Valenzuela, A.M. Allan, and K.K. Caldwell examined the role of the extracellular signal-regulated kinase (ERK1/2) signaling pathway in hippocampal-dependent learning deficits in a mouse model of FAS. Using a hippocampal slice preparation, they found that N-methyl-D-aspartate (NMDA) increased anti-phosphoERK2 immunoreactivity in control slices, but not in alcohol-exposed slices. These results may have important implications regarding alcohol-induced deficits in hippocampal-dependent learning and memory.

B.R. Monk, D.N. Linsenbardt, N.N.H. McGough, H.D. Dominguez, and J.D. Thomas hypothesized that the anti-oxidants found in pomegranate juice may protect the developing brain from oxidative injury associated with alcohol exposure. They found that pomegranate juice significantly attenuated alcohol-induced motor coordination deficits, but did not alter alcohol's effect on spatial learning. The data suggest that the antioxidant properties of pomegranate juice may protect the cerebellum and other motor areas against alcohol-induced neurotoxicity.

S. Chen, M.F. Wilkemeyer and M.E. Charness examined the effects of alcohol on NAP (octapeptide NAPVSIPQ)-mediated neurite outgrowth. Using cerebellar neurons, they found that alcohol inhibits NAP-mediated neurite extension, with significant effects at concentrations as low as $10 \mathrm{mM}$. These results suggest that alcohol induces some of its neuroteratogenic effects by interfering with peptide-mediated neuronal growth and by disrupting glial-neuronal interactions.

C. Arias and M.G. Chotro examined how prenatal alcohol exposure affects postnatal conditioning to alcohol's flavor. Using a rat model, they found that prenatal alcohol exposure delayed postnatal acquisition of a conditioned aversion to the taste of alcohol. The results 
support the hypothesis that prenatal alcohol exposure establishes an appetitive associative memory. The findings may have important implications regarding prenatal alcohol exposure and later alcohol use.

F.C. Zhou, H.J. Edenberg, C. Goodlett and L.Ni analyzed the development of hemopoiesis in cultured rodent embryos using morphological and DNA microarray analyses. They found that alcohol reduced red blood cell (RBC) content in the yolk sac and circulation and increased embryonic heart rate. In addition, alcohol reduced expression of a cohort of genes for hemopoiesis. The findings indicate that alcohol affects RBC formation in early embryonic development. The authors suggested that this effect on hemopoiesis may contribute to the growth deficits and microencephaly that accompany FAS.

\section{In the human/clinical research category}

K. Nash, E.D. Sheard, M. Williamson, M. Noseworthy, M. Desrocher and J. Rovet investigated whether metabolic brain function correlates with social function in children with FASD. They obtained magnetic resonance spectroscopy scans in children with FASD and in control children. From the children's parents, they obtained questionnaire data regarding behavior, attention, memory, and executive function. In the alcohol-exposed children, the investigators found abnormally low glutamate levels and an association between left hippocampal $\mathrm{N}$-acetylaspartate levels and parent ratings of emotional regulation. The authors concluded that the social and emotional problems in children with FASD may be related to regional oxidative stress damage.

S.L. Fryer, S.F. Tapert, S.N. Mattson, A.D. Spadoni and E.P. Riley investigated the neural basis of cognitive control impairments commonly observed in children prenatally exposed to alcohol. Alcohol-exposed and control children underwent functional MRI while performing a response inhibition task. The investigators found significantly more activation during no-go relative to go trials among alcohol-exposed children. The results suggest that children exposed to alcohol during gestation increase brain response in several cortical regions, including prefrontal areas, compared to non-exposed children. The observed patterns may indicate decreased neural efficiency while performing a cognitive task.

M. Chen, H. Carmichael Olson and S.J. Astley employed parental questionnaires and overnight polysomnography to investigate the prevalence and nature of sleep problems in children with FASD. The questionnaires revealed that large proportions (35-50\%) of the children with FASD showed abnormal amounts of sleep (primarily too little sleep), whereas none in the comparison group exhibited sleep problems. In the children with FASD, overnight polysomnography showed abnormal sleep architecture, fragmented sleep, and sleep-disordered breathing with obstructive hypoventilation. These same children had extreme daytime hyperactivity. The data suggest a high prevalence of sleep problems in children with FASD. These nocturnal sleep problems may contribute to daytime impairments in neurocognitive and behavioral performance.

M.J. Burden, S.W. Jacobson, S. Dehaene, N. Dodge and J.L. Jacobson examined the effects of prenatal alcohol and cocaine on arithmetic and "number sense." Children whose mothers' alcohol and drug use were ascertained during pregnancy were assessed on a 7-part arithmetic test. The investigators found that prenatal alcohol exposure was associated with poorer performance on both exact and approximate calculation measures, as well as for number comparison and proximity judgment. None of these effects were related to cocaine exposure. The data suggest that poorer calculation and arithmetic abilities are linked to poorer "number sense" or magnitude representation, and that these effects are associated specifically with alcohol, rather than with cocaine. 
C.M. Adnams, W. Kalberg, P.W. Kodituwakku, M.D. Perold, P. Sorour, B. Castle, S. September, R. Adams, A. Kotze, W. Rossouw, E. Williams, P. Engelbrecht, and P. May studied the effect of 18 school months of classroom or parent interventions on cognition and behavior in children with FASD in the Western Cape Province. Children with FASD or non-FASD controls were randomized to 4 groups-Cognitive Control Therapy, Language and Literacy Training, Parent Intervention or FASD control. A battery of tests, questionnaires and assessments were performed. The study is currently at its mid-stage. At the study's commencement, children with FASD had significantly worse behavior and academic performance than non-FASD controls. At mid-intervention, there were no significant gains by intervention groups over controls on core cognitive assessments. However, the classroom intervention groups did have greater improvements than controls in some cognitive areas, including reading comprehension and pre-literacy. The investigators concluded that children with FASD show cognitive improvements in some specific areas targeted by classroom interventions.

D.G. Buckley, P.A. May, D. Fiorentino, J.P. Gossage, W.O. Kalberg, H.E. Hoyme, L.K. Robinson, G. Coriale and M. Ceccanti sought to determine the prevalence of FASD in Western European populations by conducting an epidemiological study of FAS and FASD in Italian schools. In the Lazio region of Italy, the investigators randomly selected 25 schools in which 543 children underwent a detailed evaluation regarding growth parameters, dysmorphology, psychological development, behavior, and prenatal alcohol exposure. From their findings, the investigators estimate the prevalence of FAS at 3.7 to 7.4 per 1000 children. When cases of partial FAS or alcohol-related neurodevelopmental deficits were included, the estimate rose to 35 per 1000. The results suggest that FASD is more common in the western world than previously estimated.

J.R. Hutson, R. Magri, H. Suarez, H. Miguez, J. Gareri, and G. Koren utilized measured levels of fatty acid ethyl esters (FAEEs) to define the prevalence of alcohol consumption during the latter two-thirds of pregnancy in Montevideo, Uraguay. Nine hundred meconium samples were collected from two public hospitals in Montevideo, and FAEE levels were determined by gas chromatography. In their preliminary results, the investigators found that $46 \%$ of the meconium samples tested positive for FAEEs. The authors concluded that approximately half of all neonates in this population are at risk of exposure to alcohol during the latter two-thirds of pregnancy.

Each year, the FASDSG awards the Student Merit Award to one student or postdoctoral fellow for excellence in FAS-related research. The 2006 FASDSG Student Merit Award went to Julie Siegenthaler, $\mathrm{PhD}$, for her work investigating the effect of alcohol on the developing cerebral cortex. Dr. Siegenthaler conducted her research in the laboratory of Dr. Michael Miller at the Upstate Medical University of New York. Dr. Siegenthaler summarized her work in an address entitled "Regulation of corticogenesis by TGF- $\beta$ : interactions with ethanol." Dr. Siegenthaler showed that transforming growth factor (TGF- $\beta$ ) is at the fulcrum of early development, defining whether a cell continues to proliferate or begins its migration. In addition, she showed that TGF- $\beta$ is a target of ethanol and may be a key intermediary through which ethanol causes its neuroteratogenic effects.

The highest honor bestowed by the FASDSG is the Henry Rosett Award, which recognizes an individual for outstanding long-term contributions to the field of FASD. This year's recipient of the Henry Rosett Award was Faye Calhoun, DPA, Deputy Director (retired) of the National Institute on Alcohol Abuse and Alcoholism (NIAAA). Dr. Calhoun was chosen for this award in recognition of her many years of devotion to the growth of FASD basic, clinical and translational research efforts supported by the federal government. Dr. Calhoun worked tirelessly to expand international FASD research, broadened the scope of FASD programs 
across multiple federal government agencies, and played an instrumental role in establishing the Interagency Coordinating Committee on Fetal Alcohol Syndrome (ICCFAS).

In accepting the Henry Rosett Award, Dr. Calhoun delivered a lecture entitled "Addressing prenatal alcohol exposure: strategy and stealth 1996-2006." Dr. Calhoun summarized the administrative history milestones of federal involvement in FASD research. She outlined the goals of NIAAA international collaborative research on FASD and the contributions of different countries to the study of FASD. Dr. Calhoun described the multiple agencies involved in the ICCFAS and explained how the different plans, insights and priorities of these different agencies strengthen the group's effectiveness. Dr. Calhoun's address included several recommendations to investigators in the field of FASD. She recommended that investigators issue plain language summaries of applicable research results, or admit that the research was an intellectual exercise. She recommended that FASD investigators pursue research avenues that truly offer a hope of translation from bench-top to clinical trials to help for children with FASD. Finally, she recommended that researchers utilize FAS research methods as the foundation for the study of other teratogens.

As always, the 2006 annual meeting of the FASDSG played an important role in advancing FASD-related research. It brought together clinicians, biologists, social scientists, psychologists, and others interested in the diagnosis, prevention, treatment, and biology of FASD. The meeting provided these individuals with the most current data regarding FASD and related topics, fostered the development of young investigators entering the field of FASD, and helped elucidate the most important gaps in the understanding of alcohol's effects on the fetus.

\section{Acknowledgements}

The annual meetings of the Fetal Alcohol Spectrum Disorders Study Group are supported by grant R13-AA015661 from the National Institute on Alcohol Abuse and Alcoholism. 